\title{
Prevalência de sífilis e HIV utilizando testes rápidos em parturientes atendidas nas maternidades públicas de Vitória, Estado do Espírito Santo
}

\author{
Prevalence of syphilis and HIV using rapid tests among parturients attended in \\ public maternity hospitals in Vitória, State of Espírito Santo
}

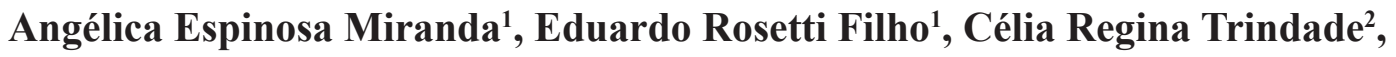 \\ Gabriel Moulin Gouvêa ${ }^{1}$, Débora Miranda $\operatorname{Costa}^{1}$, Thales Ge Oliveira ${ }^{1}$, \\ Luis Cláudio França ${ }^{3}$ e Reynaldo Dietze ${ }^{1}$
}

\begin{abstract}
RESUMO
O objetivo deste estudo foi descrever a prevalência de sífilis e HIV em parturientes atendidas nas maternidades públicas, Vitória, ES. No período de janeiro a maio 2007, elas responderam a entrevista contendo dados sócio-demográficos, comportamentais e clínicos e realizaram teste rápido, ELISA e imunofluorescência indireta para HIV; e teste rápido, VDRL e MHA-TP para sífilis. Um total de 1.380 parturientes foi incluído. A média de idade foi 24,2 (DP 6,1) anos e escolaridade 8,5 (DP 2,6) anos. A prevalência de HIV foi 0,6\% (IC95\% 0,2\%-1,1\%) e sífilis 0,4\% (IC95\% 0,2\%-0,9\%). 0 teste rápido para HIV foi concordante com o ELISA e a imunofluorescência indireta em todos os casos. 0 teste rápido para sífilis foi positivo em seis parturientes, sendo que dois resultados não foram confirmados pelo VDRL e MHA-TP. Entre 71 (5,1\%) parturientes que não realizaram pré-natal, o teste rápido para sífilis foi positivo em uma e o HIV em duas delas. Os resultados indicam a importância do teste rápido para o diagnóstico de sífilis e HIV, pois há parturientes que não realizam pré-natal ou que não tem acesso ao resultado ou ao tratamento durante o pré-natal.
\end{abstract}

Palavras-chaves: Testes rápidos. Gestação. Sífilis. Vírus da imunodeficiência humana. Fatores de risco.

\section{ABSTRACT}

The aim of this study was to describe the prevalence of syphilis and HIV among parturients attended at public maternity hospitals in Vitória, Espírito Santo. Between January and May 2007, interviews were conducted to obtain demographic, behavioral and clinical data. The subjects were tested for HIV using a rapid test, ELISA and the indirect fluorescence assay; and for syphilis using a rapid test, VDRL and MHA-TP. A total of 1,380 women were included. Their mean age was 24.2 years (SD 6.1) and their mean schooling level was 8.5 years (SD 2.6). The HIV prevalence rate was $0.6 \%$ (95\% CI: $0.2 \%-1.1 \%$ ) and the syphilis rate was $0.4 \%$ (95\% CI: $0.2 \%-0.9 \%$ ). The rapid test for HIV was in agreement with ELISA and the indirect fluorescence assay in all cases. The rapid test for syphilis was positive in six women, but two cases were not confirmed by VDRL and MHA-TP. Among the $71(5.1 \%)$ women who had not had antenatal care, the rapid test on one woman was positive for syphilis and two for HIV. The results show the importance of the rapid test for diagnosing syphilis and HIV because there are parturients without antenatal care or without access to test results and treatment during antenatal care.

Key-words: Rapid tests. Pregnancy. Syphilis. Human immunodeficiency virus. Risk factors.

A falha na detecção precoce da infecção pelo vírus da imunodeficiência humana (HIV) durante o pré-natal representa uma oportunidade perdida de intervenção na gestante infectada, limitando as possibilidades de redução da incidência dos casos pediátricos infectados por transmissão materno-infantil. Grande parte destas mulheres não tem acesso ao teste de HIV, seja pela

1. Departamento de Medicina Social, Núcleo de Doenças Infecciosas, Universidade Federal do Espírito Santo, Vitória, ES. 2. Departamento de Obstetrícia, Escola de Medicina da Santa Casa de Misericórdia de Vitória, Vitória, ES. 3. Departamento de Ginecologia e Obstetrícia, Universidade Federal do Espírito Santo, Vitória, ES. Endereço para correspondência: Dra. Angélica Espinosa Miranda. Núcleo de Doenças Infecciosas/UFES. Avenida Marechal Campos 1468, Maruípe, 29040-091 Vitória, ES

e-mail: espinosa@ndi.ufes.br

Tel: 5527 3335-7210

Recebido para publicação em 14/04/2009

Aceito em 11/08/2009 sua condição social, seja por falhas no sistema de saúde ${ }^{23} 24$. Mulheres vivendo com a infecção pelo HIV constituem a principal fonte de infecção em crianças, 95,1\% dos casos de síndrome da imunodeficiência adquirida (AIDS) em indivíduos menores de 14 anos no Brasil, até junho de 2008, foram casos de transmissão materno-infantil. Em 2007, foram notificadas 6.017 gestantes com sorologia positiva para HIV, sendo 104 casos no Estado do Espírito Santo ${ }^{21}$.

O Ministério da Saúde recomenda que o teste da sífilis seja oferecido a todas as gestantes nos primeiros estágios da gravidez e tem realizado campanhas para a eliminação da sífilis congênita no país ${ }^{19}{ }^{20}$. Entretanto, a sífilis permanece como agravo comum na gestação, apesar de testes diagnósticos baratos e acurados estarem disponíveis no mercado e da persistente sensibilidade do Treponema pallidum à penicilina ${ }^{320}$. Em 2007, foram notificados ao Ministério da Saúde 6.143 casos de sífilis em gestantes e 
1.004 casos de sífilis congênita ${ }^{21}$. No ano de 2006, entre os casos notificados de crianças com sífilis congênita, 78,7\% das mães tinham história de ter feito pré-natal e destas 43,6\% detectaram a sífilis na ocasião e em somente $23,6 \%$ dos casos o parceiro sexual foi tratado ${ }^{20}$.

A utilização dos testes rápidos, para o diagnóstico de infecção pelo HIV e da sífilis na gestação, tem se mostrado uma estratégia eficaz na implementação de novas medidas de abordagem para o diagnóstico rápido e eficaz dessas infecções durante a assistência ao pré-natal e ao parto $^{710}$. 0 teste rápido para HIV faz parte da rotina nas maternidades brasileiras, apesar de nem sempre estar disponível, já o teste rápido para sífilis ainda precisa ser implementado a nível nacional.

Os testes rápidos são mais importantes em áreas com baixa adesão ao pré-natal e poucos recursos diagnósticos. Apesar de Vitória ser um município com alta taxa de atenção ao pré-natal, não é conhecido o quanto que a realização dos testes rápidos nas maternidades públicas poderia implementar o diagnóstico dessas infecções. Para tanto o objetivo deste estudo foi descrever a prevalência de sífilis e HIV em parturientes atendidas nas maternidades públicas do Município de Vitória, ES.

\section{MATERIAL E MÉTODOS}

População de estudo. Estudo descritivo em corte transversal realizado em parturientes que buscaram atendimento nas três maternidades públicas de Vitória, maternidades de baixo, médio e alto risco. Todas as parturientes, que procuraram os serviços de assistência ao parto, no período de janeiro a maio de 2007, foram convidadas a participar do estudo.

Coleta de dados. Entrevista face-a-face contendo dados sócio-demográficos; dados clínicos; sexuais; e comportamentais de risco para doenças sexualmente transmissíveis (DST) foi realizada após assinatura do termo de consentimento esclarecido.

$\mathrm{Na}$ internação, foram realizados testes rápidos para HIV (Laboratório Núcleo de Doenças Infecciosas, UFES) e para sífilis (Determine, Laboratório ABBOTT) de acordo com as recomendações dos fabricantes. 0 venereal disease research laboratory (VDRL) (Laboratórios Laborclin) também foi realizado com parte da rotina em todas as parturientes. Os testes rápidos positivos para HIV foram confirmados pelo teste Enzymelinked immunosorbent assay (ELISA) (HIV-1 Microelisa, Organon Laboratories) e pela imunofluorescência indireta (IFI) (HIV-1 IFA, FIOCRUZ). A microhemoaglutinação-Treponema pallidum (MHA-TP, Laboratórios ABBOTT) foi realizada em todos os casos com teste rápido positivo para sífilis, VDRL positivo ou discordância entre os dois testes.

Cálculo de tamanho da amostra. No Município de Vitória são atendidas, aproximadamente, 4.200 gestantes/ano, sendo que em média 3.066 são atendidas no serviço público de saúde ${ }^{2}$. 0 tamanho da amostra foi calculado para estimar a taxa de prevalência de infecção pelo HIV na população de parturientes atendidas no Município de Vitória, com um intervalo de $95 \%$ de confiança de tamanho bilateral de 0,5\%. Tomou-se como base para cálculo uma taxa de infecção na gestação de aproximadamente $1 \%{ }^{12}$, o que gerou um número de 1.137 gestantes. Incluindo uma perda de aproximadamente 20\%, o tamanho de amostra final foi de 1.500 gestantes, o que representa $42,7 \%$ das gestantes atendidas anualmente no serviço público de saúde do Município. As parturientes foram selecionadas proporcionalmente, segundo número de atendimento nas três maternidades públicas de Vitória.

Análise estatística. A análise estatística foi realizada utilizando-se o SPSS versão 11.5 para Windows. Foi realizada uma análise descritiva, incluindo distribuição de frequência para variáveis qualitativas e cálculo de média e desvio-padrão para variáveis quantitativas. As taxas de prevalência de infecção pelo HIV e sífilis foram estimadas pela presença de teste confirmatório positivo e foram fornecidas pelo diagnóstico em questão, sendo calculados os correspondentes intervalos de confiança a 95\%. As possíveis associações entre as infecções e comportamentos de risco ou variáveis demográficas e clínicas foram testadas através de testes de qui-quadrado com correção de Yates ou de Fischer quando apropriado. Odds ratio e intervalos de confiança foram calculados em análises bivariadas para estimar o grau de associação entre cada infecção e os potenciais fatores de risco. Análise multivariada de regressão logística para estimar o efeito de uma variável, ao mesmo tempo em que se controla o efeito das demais, na probabilidade de apresentar sífilis ou HIV na gravidez foi aplicada.

Aspectos éticos. Este projeto foi submetido ao Comitê de Ética em Pesquisa do Centro de Ciências da Saúde da Universidade Federal do Espírito Santo (CEP-CCS-UFES). Todas as parturientes foram convidadas a participar do projeto em caráter voluntário. Elas receberam aconselhamento e tratamento, de acordo com as normas do manual de controle das doenças sexualmente transmissíveis do ministério da saúde, para as infecções diagnosticadas $^{19}$.

\section{RESULTADOS}

Um total de 1.380 (92\%) parturientes participou do estudo das 1.500 inicialmente selecionadas. A média de idade foi de 24,2 (DP 6,1) anos, a média de escolaridade foi de 8,5 (DP 2,6) anos de estudo. A prevalência de HIV foi de 0,6\% (IC95\% 0,2\%-1,1\%) e a se sífilis foi de $0,4 \%$ (IC95\% 0,2\%-0,9\%).

0 teste rápido para o HIV foi concordante com o ELISA e a IFI em todos os casos. 0 teste rápido para sífilis foi positivo em seis parturientes sendo que destes dois não foram confirmados pelo VDRL e MHA-T'P. Um dos testes positivos confirmatórios para sífilis teve o teste rápido negativo. Entre as $71(5,1 \%)$ parturientes que não tinham realizado pré-natal, e portanto não tinham resultados de exames, o teste rápido para sífilis foi positivo em uma e o de HIV positivo em duas delas.

Os testes para diagnóstico da infecção pelo HIV e o VDRL para diagnóstico da sífilis não foram realizados ou não foram entregues os resultados em 10,3\% das gestantes que fizeram o pré-natal. 
Entre as gestantes que fizeram o teste no pré-natal, o VDRL foi positivo em 14 (1\%) gestantes, sendo que entre estas 50\% dos parceiros também fizeram o VDRL e 42,8\% destes receberam tratamento. 0 resultado do HbsAg estava no cartão de pré-natal de 1.198 parturientes, nessas parturientes a prevalência de infecção crônica pelo vírus B foi de 0,4\% (IC95\% 0,36\%-0,44\%).

A média de idade da primeira relação sexual foi de 16,5 (DP 2,9) anos. A mediana da idade gestacional foi 39 (DIQ 37; 40) semanas. Os dados sócio-demográficos estão descritos na Tabela 1. Mais de 25\% das gestantes eram adolescentes; $83,9 \%$ tinham renda familiar de até três salários mínimos e 55,7\% tiveram partos normais. Aproximadamente, $95 \%$ das parturientes relataram ter feito pré-natal, sendo que entre estas $8,5 \%$ relatou menos de quatro consultas e somente $54,8 \%$ realizou seis ou mais consultas. 0s fatores de risco relatados foram: história de DST $(4,1 \%)$, transfusão sanguínea $(3,2 \%)$, drogas injetáveis $(0,9 \%)$, drogas não injetáveis $(3,8 \%)$ e prostituição $(0,3 \%)$; $89,3 \%$ relataram parceiro único no último ano. História de DST e prostituição foram fatores de risco tanto para a infecção pelo HIV e como para a sífilis; A realização de pré-natal e ter parceiro único no último ano foram fatores de proteção para as infecções. 0 uso de drogas não injetáveis, ter parceiro com sorologia positiva para HIV e com história de encarceramento estiveram associados com HIV e a história de violência sexual com a sífilis (Tabela 2).

0 modelo final de regressão logística não mostrou associação estatística entre as infecções e os fatores de risco estudados.

TABELA 1

Características socio-demográficas das 1.380 parturientes atendidas nas maternidades públicas de Vitória, ES.

\begin{tabular}{|c|c|c|}
\hline Variáveis & Número & Percentagem \\
\hline \multicolumn{3}{|l|}{ Estado civil } \\
\hline solteira & 289 & 20,9 \\
\hline casada/união estável & 1.071 & 77,6 \\
\hline divorciada/separada & 18 & 1,3 \\
\hline viúva & 2 & 0,1 \\
\hline \multicolumn{3}{|l|}{ Idade (anos) } \\
\hline $13-19$ & 352 & 25,5 \\
\hline $20-29$ & 778 & 56,4 \\
\hline $30-39$ & 224 & 16,2 \\
\hline $40-44$ & 26 & 1,9 \\
\hline \multicolumn{3}{|l|}{ Maternidade } \\
\hline Pro-Matre (baixo risco) & 933 & 67,7 \\
\hline HUCAM (alto risco) & 289 & 21,0 \\
\hline Santa Casa (médio risco) & 157 & 11,4 \\
\hline \multicolumn{3}{|c|}{ Renda familiar em salários mínimos } \\
\hline$<1$ & 439 & 31,8 \\
\hline 1 a 3 & 719 & 52,1 \\
\hline 3 a 5 & 177 & 12,8 \\
\hline$>5$ & 45 & 3,3 \\
\hline \multicolumn{3}{|l|}{ Motivo de Internação } \\
\hline parto normal & 769 & 55,7 \\
\hline parto cesariana & 454 & 32,9 \\
\hline abortamento & 51 & 3,7 \\
\hline tratamento Clínico & 106 & 7,7 \\
\hline
\end{tabular}

TABELA 2

Frequência dos fatores de risco relatados pelas 1.380 parturientes e suas associações com a infecção pelo HIV e sífilis.

\begin{tabular}{|c|c|c|c|}
\hline & & *OR (IC 95\%) & *OR (IC 95\%) \\
\hline Variáveis & Percentagem & HIV & Sífilis \\
\hline Realizou pré-natal & 94,8 & $0,16(0,03-0,80)$ & $0,22(0,03-1,95)$ \\
\hline História de DST & 4,1 & $14,64(3,41-62,86)$ & $49,85(8,93-278,23)$ \\
\hline Uso drogas injetáveis & 0,9 & $1,01(1,00-1,01)$ & $* *$ \\
\hline Usuária drogas não-injetáveis & 3,8 & $8,81(1,74-44,76)$ & $5,19(0,60-45,22)$ \\
\hline História de prostituição & 0,3 & $228,33(27,47-1435,84)$ & $91,40(8,07-1035,67)$ \\
\hline História de violência sexual & 2,0 & $7,12(0,85-59,86)$ & $9,98(1,35-88,32)$ \\
\hline Parceiro com história de encarceramento & 4,3 & $7,69(1,52-38,94)$ & $5,68(0,63-51,59)$ \\
\hline Parceiro único no último ano & 89,3 & $0,12(0,03-0,47)$ & $0,12(0,023-0,58)$ \\
\hline Parceiro portador do vírus HIV & 0,9 & $170,50(36,17-803,62)$ & $* *$ \\
\hline
\end{tabular}

* OR (IC 95\%): Odds ratio com intervalo de confiança de 95\%

** 0 cálculo da associação não pode ser realizado na ausência de células vazias, DST: doença sexualmente transmissível, HIV: vírus da imunodeficiência humana. 


\section{DISCUSSÃo}

Este estudo, que teve como finalidade descrever a prevalência de HIV e sífilis em parturientes atendidas nas maternidades públicas, identificou que o teste rápido foi importante na abordagem das parturientes que não tinham realizado pré-natal $(5,1 \%)$, e portanto não tinham resultados de exames. 0 teste rápido diagnosticou um caso de sífilis e dois casos de infecção pelo HIV possibilitando o tratamento dessas mulheres ainda na maternidade.

A Organização Mundial de Saúde (OMS) tem uma estratégia que estimula a pesquisa de novos testes rápidos que, tenham como requisitos o resultado rápido (menos de 15 minutos), sejam fáceis de usar por profissionais de saúde de área básica, não necessitem recursos laboratoriais tradicionais, sejam estáveis à temperatura ambiente, possuam boa sensibilidade e especificidade e baixo custo. Com essas características, os testes podem ser utilizados em larga escala nos serviços de assistência primária de saúde como o Programa de Saúde da Família e também em maternidades ${ }^{14}$.

As taxas de prevalência de infecção pelo HIV, encontradas neste estudo, foram semelhantes à prevalência de $0,4 \%$ relatada no Estudo-Sentinela de 2004. Em relação à sífilis, entretanto, a taxa de prevalência foi mais baixa uma vez que o estudo sentinela relatou uma taxa de 1,6\% de VDRL positivo ${ }^{23}$. Outros estudos realizados no Brasil mostram que as taxas de prevalência de HIV não têm apresentado variação significante nas últimas décadas ${ }^{6} 1012131522$. Já as taxas de sífilis variavam de 2,4\% a 5,2\% na década de $1990^{312}{ }^{17}$, com exceção a de Amaral e cols ${ }^{1}$, que já relatavam uma taxa mais baixa (1,2\%), em Campinas. A partir de 2001, os trabalhos identificaram taxas de prevalência mais baixas em gestantes variando de $0,8 \%$ a $1,6 \%{ }^{410131516}$. Desta forma, o resultado encontrado neste estudo, $1 \%$ no cartão pré-natal e $0,4 \%$ no momento do parto, está em concordância com a tendência de queda das taxas de prevalência de sífilis no Brasil ocasionada pela implementação das campanhas do Ministério da saúde direcionadas a orientar os profissionais de saúde e as gestantes em relação aos riscos da síflis congênita e a necessidade do diagnóstico e tratamento precoce durante o prénatal $^{19}$. Os dados de infecção crônica pelo vírus B relatados neste estudo estão de acordo com os encontrados em outros estudos realizados no Brasil $^{10} 1215$.

Este estudo encontrou uma cobertura de sorologia para HIV na gestação de 89,7\%. 0 Estudo Sentinela Parturiente (2004) estimou a cobertura nacional de $63 \%$, sendo que na região Sudeste a cobertura foi de $78,3 \%{ }^{23}$. A falha nessa cobertura pode ser explicada por falhas no processo de detecção da infecção pelo HIV durante a gestação, destacando-se a ausência de pré-natal em 4,1\% dos casos durante o pré-natal, a ausência de pedido do teste em $21 \%$, a recusa da gestante em $3,4 \%$ e desconhecimento do resultado no parto em $9 \%{ }^{23}$. Esses fatores ajudam a explicar a manutenção das taxas de transmissão vertical do HIV, apesar das intervenções preconizadas pelo Programa Nacional de DST e Aids serem reconhecidamente efetivas.

Neste estudo, houve discordância entre os resultados do VDRL e do teste rápido para sífilis em alguns casos. Os testes disponíveis nas maternidades, e comumente utilizados para 0 diagnóstico da sífilis no Brasil, são os testes não treponêmicos, sendo o VDRL o mais utilizado. Além do rastreamento, ele permite o acompanhamento do paciente através das quedas de titulação ao longo do tempo, após ter sido realizado o tratamento, mas necessita de um teste treponêmico para confirmação do resultado ${ }^{9}$. 0 desenvolvimento de testes rápidos para sífilis que sejam treponêmicos e não treponêmicos é importante para 0 diagnóstico desses casos e para a implementação da assistência durante o pré-natal e o parto.

Entre as gestantes que fizeram acompanhamento pré-natal, mais de $10 \%$ não fizeram nem mesmo o VDRL, ou se fizeram e não tiveram acesso ao resultado do exame. 0 início tardio do acompanhamento pré-natal e a demora na tramitação dos exames de rotina favorecem estes índices de falha na prevenção e no diagnóstico durante o pré-natal ${ }^{192} 23$, o que enfatiza a importância da utilização do teste rápido para o diagnóstico desses casos. De modo geral, pelo excesso de demanda para uma infra-estrutura deficitária, o período decorrido entre a solicitação do exame, 0 agendamento para colher o sangue e a disponibilidade do resultado gira em torno de 30-60 dias ${ }^{1620}$. A avaliação da qualidade do pré-natal com a realização do teste para sífilis (VDRL) no primeiro e terceiro trimestre de gestação e na hora do parto, o tratamento adequado da mãe e do parceiro e as notificações dos casos, são essenciais para que se possa controlar as taxas de infecção materno-infantil ${ }^{20} 23$.

Apesar da alta taxa de realização do pré-natal, quando se estratifica pelo número de consultas realizadas pode-se notar que a assistência ainda pode ser melhorada, pois apesar de mais de $90 \%$ relatado a realização de quatro ou mais consultas somente $54,8 \%$ relatou seis ou mais consultas. A recomendação da OMS é de que sejam realizadas pelo menos 4 consultas para acompanhamento pré-natal. No Brasil, o Ministério da Saúde, de acordo com a Portaria de número 570 de 01/06/2000, considera um mínimo de seis consultas para o período pré-natal ${ }^{18}$.

A maioria das parturientes não soube informar sobre a sorologia dos parceiros sexuais; entre aquelas com resultado de VDRL positivo, somente $50 \%$ delas conseguiram trazer o parceiro para participar do pré-natal e ser orientado e um percentual ainda menor conseguiu que o parceiro fosse testado, e entre estes nem todos concordaram com o tratamento. As falhas de atendimento, diagnóstico e tratamento das gestantes são agravadas de modo importante pela elevada taxa de ausência do parceiro nesta abordagem. Não há como esperar tratamento e cura da sífilis e controle da infecção pelo HIV na gestação quando um grande número de parceiros não é abordado pelo serviço de saúde, não se submete a exames para diagnóstico de sífilis e/ ou outras doenças de transmissão sexual, não realiza tratamento adequado e não faz exame laboratorial para controle de tratamento nos moldes preconizados para a gestante ${ }^{8}$. Com isto, as gestantes portadoras dessas infecções vivenciam a ineficiência do tratamento, a re-infecção e a transmissão vertical.

História prévia de DST e prostituição foram os fatores que, na análise bivariada, se associaram com infecção pelo HIV e sífilis neste estudo. Outros estudos realizados em Vitória e no Brasil também demonstraram a importância do comportamento sexual, como 0 não uso do preservativo, a história prévia de DST e prostituição, na presença da infecção pelo HIV e de outras DST em gestantes ${ }^{45912}$. 
Embora o estudo transversal não seja o ideal na avaliação do uso dos testes rápidos, sua aplicação se justifica porque, implementar a assistência à saúde das gestantes é importante para demonstrar a susceptibilidade da população a infecções que causam complicações no ciclo gravídico-puerperal. Devido à baixa prevalência das infecções estudadas e dos fatores de risco, o tamanho da amostra não foi suficiente para encontrar uma forte associação estatística entre conhecidos fatores de risco e as infecções. A possibilidade de ter ocorrido viés de resposta não pode ser descartada devido à tendência de se dar respostas socialmente aceitáveis. A falta de acurácia a respeito do uso de preservativo, idade da primeira relação sexual, número de parceiros e uso de drogas, entre outras, não podem ser excluídas.

Os resultados deste estudo indicam a importância da realização do teste rápido HIV e do VDRL na maternidade, uma vez que há parturientes que não realizam pré-natal e há aquelas que apesar de realizar, não tem acesso ao resultado e ou ao tratamento durante o pré-natal. Isso significa dizer que cerca de 71 (5,1\%) gestantes em Vitória que não foram captadas na gestação deixariam de ter acesso ao diagnóstico, de receber tratamento e controle da infecção quando necessários, se não fossem testadas na maternidade. No caso da sífilis, observou-se que apesar da disponibilidade de recursos profiláticos baratos e eficientes para redução da transmissão materno-infantil, 10,3\% das mulheres foram excluídas, já que não realizaram ou não tiveram acesso ao resultado do teste de sífilis no pré-natal e, portanto, não teriam sido tratadas. Entretanto, para a implementação do teste rápido para sífilis nas maternidades é necessário haver a recomendação pelo Ministério da Saúde.

A erradicação da sífilis congênita e o controle da transmissão vertical do HIV só serão possíveis quando se priorizar o diagnóstico precoce e assegurar o tratamento da mulher jovem, especialmente durante a gestação, e a utilização dos testes rápidos pode agilizar o diagnóstico principalmente em áreas com recursos diagnósticos limitados.

\section{AGRADECIMENTOS}

Os autores agradecem ao Dr. Valdir Monteiro Pinto e Drẩ Lílian Amaral Inocêncio, do Programa Nacional de DST e Aids, Ministério da Saúde do Brasil, pela doação dos testes rápidos para detecção de sífilis e HIV. Agradecem também aos diretores das três maternidades públicas do Município de Vitória que autorizaram a realização da pesquisa.

\section{REFERÊNCIAS}

1. Amaral E, Faundes A, Goncales NS, Paula CA. Prevalence of HIV and Treponema pallidum infections in pregnant women in Campinas and their association with sociodemographic factors. Revista Paulista de Medicina 114:1108-1116, 1996.

2. Banco de dados do Sistema Único de saúde (DATASUS) [homepage on the internet]. Brasil: [cited 10 ago. 2005]. Acesso em 20 de março de 2008. Disponível em: http://www.datasus.gov.br/indicadores.
3. Barsanti C, Diniz EMA, Succi RCM. Diagnóstico de sífilis congênita: comparação entre testes sorógicos na mãe e no recém-nascido. Revista da Sociedade Brasileira de Medicina Tropical 32: 605-611, 1999.

4. Benzaken AS, García EG, Rodrigues AO, Mora C, Sanchez MS, Sardinha JC. "Madre Fronteriza": estudo conjunto de prevalência de sífilis tricomoníase e HIV em gestantes da tríplice fronteira do Alto Rio Solimões. DST. Jornal Brasileiro de Doenças Sexualmente Transmissíveis 16:15-23, 2004.

5. Calvet, GA, Custódio-João E, Nielsen-Saines K, Cunha CB, Menezes JA, D'Ippolito MM, Santos-Cruz ML, Martins EB, Santos-Silva SM, Medeiros AF, Matos HJ. Tendências temporais observadas em uma coorte de gestantes infectadas pelo HIV acompanhadas no Rio de Janeiro de 1996 a 2004. Revista Brasileira de Epidemiologia 10: 323-337, 2007.

6. Cardoso AJ, Griep RH, Carvalho HB, Barros A, Silva SB, Remien R. Infecção pelo HIV entre gestantes atendidas nos centros de testagem e aconselhamento em Aids. Revista de Saúde Pública 41:101-108, 2007.

7. Dennis RL, Negron TJ, Lindsay M, Nesheim SR, Lee FK, Jamieson DJ. Rapid human immunodeficiency virus testing in labor and delivery: a comparison of implementation models between 2 hospitals. The Journal of Perinatal and Neonatal Nursing 21:298-306, 2007.

8. Duarte G. Extensão da assistência pré-natal ao parceiro como estratégia de aumento da adesão ao pré-natal e redução da transmissão vertical de infecções. Revista Brasileira de Ginecologia e Obstetrícia 29: 171-174, 2007.

9. Ebel A, Bachelart L, Alonso JM. Evaluation of a new competitive immunoassay (BioElisa Syphilis) for screening for Treponema pallidum antibodies at various stages of syphilis. Journal of Clinical Microbiology 36:358-61, 1998.

10. Figueiró-Filho EA, Senefonte FR, Lopes AH, de Morais 00, Souza Júnior VG, Maia TL, Duarte G. Frequency of HIV-1, rubella, syphilis, toxoplasmosis, cytomegalovirus, simple herpes virus, hepatitis B, hepatitis $\mathrm{C}$, Chagas disease and HTLV I/II infection in pregnant women of State of Mato Grosso do Sul. Revista da Sociedade Brasileira de Medicina Tropical 40: 181-187, 2007.

11. Mabey D, Peeling RW, Ballard R, Benzaken AS, Galbán E, Changalucha J, Everett D, Balira R, Fitzgerald D, Joseph P, Nerette S, Li J, Zheng H.. Prospective, multi-centre clinic-based evaluation of four rapid diagnostic tests for syphilis. Sexually Transmitted Infection 82 (S5):v13-6, 2006.

12. Miranda AE, Alves MC, Neto RL, Areal KR, Gerbase AC. Seroprevalence of HIV, hepatitis B virus, and syphilis in women at their first visit to public antenatal clinics in Vitória, Brazil. Sexually Transmitted Diseases 28: 710-713, 2001.

13. Olbrich Neto J, Meira DA. Soroprevalência de vírus linfotrópico de células T humanas, vírus da imunodeficiência humana, sífilis e toxoplasmose em gestantes de Botucatu - São Paulo, Brasil. Fatores de Risco para vírus linfotrópico de células T humanas. Revista da Sociedade Brasileira de Medicina Tropical 37:28-32, 2004.

14. Peeling RW, Htun Ye. Diagnostic tools for preventing and management maternal and congenital syphilis: and overview. Bulletin of World Health Organization 82:439-446, 2004.

15. Reiche EM, Morimoto HK, Farias GN, Hisatsugu KR, Geller L, Gomes AC, Inoue HY, Rodrigues G, Matsuo T. Prevalência de tripanossomíase americana, sífilis, toxoplasmose, rubéola, hepatite $\mathrm{B}$, hepatite $\mathrm{C}$ e da infecção pelo vírus da imunodeficiência humana, avaliada por intermédio de testes sorológicos, em gestantes atendidas no período de 1996 a 1998 no Hospital Universitário Regional Norte do Paraná. Revista da Sociedade Brasileira de Medicina Tropical 33:519-527, 2000.

16. Rodrigues CS, Guimarães MDG; Grupo Nacional de Estudo sobre Sífilis Congênita. Positividade para sífilis em puérperas: ainda um desafio para o Brasil. Revista Panamericana de Saúde Pública 16:168-175, 2004.

17. Santos JI, Lopes MA, Deliege-Vasconcelos E, Couto-Fernandez JC, Patel BN, Barreto ML, Ferreira Júnior OC, Galvão-Castro B. Seroprevalence of HIV, HTLV-I/ II and other perinatally transmitted pathogens in Salvador, Bahia. Revista do Instituto de Medicina Tropical de São Paulo 37: 343-348, 1995.

18. Secretaria de Vigilância em Saúde. Portaria número 570. Publicada no Diário Oficial (DO-110-E de 08/06/2000). Ministério da Saúde, Brasília, 2000.

19. Secretaria de Vigilância em Saúde. Programa Nacional de DST e Aids. Manual de Controle das Doenças Sexualmente Transmissíveis. Ministério da Saúde, Brasília, $n^{0}$ 68, 2006.

20. Secretaria de Vigilância em Saúde. Boletim Epidemiológico eletrônico. Avaliação da notificação da sífilis congênita no Brasil, 2000 A 2003. Ano 07, NO 07, Ministério da Saúde, Brasília, 2007. 
21. Secretaria de Vigilância em Saúde. Programa Nacional de DST e Aids. Boletim Epidemiológico AIDS e DST. 2008, Ano $\mathrm{V} \mathrm{n}{ }^{0} 01-27^{\mathrm{a}}$ a $52^{\mathrm{a}}$ semanas epidemiológicas - julho a dezembro de $2007-01^{\mathrm{a}} \mathrm{a} 26^{\mathrm{a}}$ semanas epidemiológicas - janeiro a junho de 2008, Ministério da Saúde, Brasília, 2008.

22. Souza NCN, Botelho CAO, Honer MR. Retrospective study of a pioneer antenatal screening program with 8477 pregnant women in Brazil. Clinical \& Experimental Obstetrics \& Gynecology 31: 217-220, 2004.
23. Souza-Júnior PRB, Szwarcwald CL, Barbosa Júnior A, Carvalho MF, Castilho EA. Infecção pelo HIV durante a gestação: Estudo-Sentinela Parturiente, Brasil, 2002. Revista de Saúde Pública 38: 764-772, 2004.

24. Szwarcwald CL, Souza Júnior PRB. Estimativa da prevalência de HIV na população brasileira de 15 a 49 anos, 2004. Boletim Epidemiológico AIDS v. III p.11-15, 2006 